\title{
Fuzzy based Low Contrast Image Enhancement Technique by using Pal and King Method
}

\author{
Ajay Kumar Gupta \\ Research Scholar \\ LNCT, Kalchuri Nagar, \\ Raisen Road Bhopal- 462021 \\ India
}

\author{
Siddharth Singh Chouhan \\ Asst. Prof., IT Dept. \\ LNCT, Kalchuri Nagar, \\ Raisen Road Bhopal- 462021 \\ India
}

\author{
Manish Shrivastava \\ Head of IT Dept. \\ LNCT, Kalchuri Nagar, \\ Raisen Road Bhopal- 462021 \\ India
}

\begin{abstract}
One of the most interesting and challenging area in image processing research is to enhance low Contrast images. Many images may suffer from poor contrast and noise due to the inadequate lighting during image acquiring. So it is required to enhance the contrast of image as well as remove the noise that decreases image quality. This paper presents a fuzzy based enhancement technique for low contrast grayscale image. Proposed works transforms the gray scale image from spatial domain to fuzzy domain, then modify the fuzzy domain by using the pal king membership function which modify image from low contrast to high contrast, And finally, transforms the gray scale image from modified fuzzy domain back to spatial domain by using defuzzification method. The performances of the proposed method are compared with the other existing methods. The proposed method gives better quality enhanced image and needs minimum processing time rather than the other methods.
\end{abstract}

\section{General Terms}

Image Enhancement, Grayscale.

\section{Keywords}

Defuzzification, Fuzzy domain, Membership Function, Spatial Domain.

\section{INTRODUCTION}

Many important areas like machine vision, remote sensing, dynamic and traffic scene analysis, biomedical image analysis and autonomous navigation required good contrast images with preserving details [1]. But most of the images suffer from poor/low contrast because of inadequate lighting during image acquiring, it may be by wrong setting of aperture size or due to speed of shutter or it may be by nonlinear image intensities mapping. Thus, image enhancement is required whenever one wants to increase the quality of the image. So Image enhancement is one of the fundamental task applied in image processing that improve interpretability and appearance of the image. It provides better input image for further image processing task [2].

Image enhancement can be used in many fields where images are to be analyzed such as satellites images analysis, medical image analysis etc. It can be defined the Image enhancement technique as transforming an image $\mathrm{X}$ into image $\mathrm{Y}$ using transformation function $T$. The values of pixels in images $X$ and $\mathrm{Y}$ are denoted by $\mathrm{x}$ and $\mathrm{y}$, respectively. As said, the pixel values $\mathrm{x}$ and $\mathrm{y}$ are related by the expression,

$$
\mathrm{y}=\mathrm{T}(\mathrm{x})
$$

Where $\mathrm{T}$ is a transformation algorithm that converts a pixel value $\mathrm{x}$ into a pixel value $\mathrm{y}$. The results of this transformation are converted into the grey scale range or color image. If grey scale digital images are used then, the results are mapped back into the range $[0, \mathrm{~L}-1]$, where $\mathrm{L}=2 \mathrm{k}, \mathrm{k}$ is the number of bits in the image being considered. So, for example, suppose if it is used an 8-bit image then the range of pixel values lie between 0 and 255. This theory can be extended for the color images also [3].

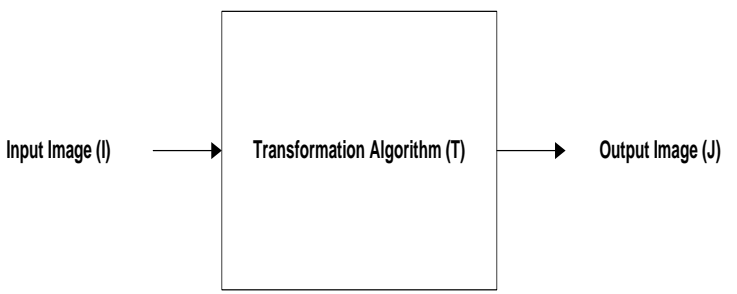

Fig 1: Image Enhancement technique

In next section, are presenting the research work of some prominent authors in the same field and explaining a short description of various techniques used for image enhancement.

\section{LITERATURE REVIEW}

Image enhancement can be classified into two groups namely frequency domain and spatial domain methods. In the frequency domain method, the enhancement is conducted by modifying the frequency transform of the image. Meanwhile in the latter method, image pixels are directly modified to enhance the image. However, computing the enhancement in frequency domain is time consuming process even with fast transformation technique thus made it unsuitable for real time application [4].

There are many image enhancement methods have been proposed. A very popular technique for image enhancement is histogram equalization (HE). This technique is commonly employed for image enhancement because of its simplicity and comparatively better performance on almost all types of images. The operation of HE is performed by remapping the gray levels of the image based on the probability distribution of the input gray levels. It stretches the dynamic range of the image's histogram and resulting in overall contrast enhancement [5]. Various researches have been performed on Histogram Equalization, and many methods have already been proposed. But Most of the HE techniques could cause a washed-out effect on the appearance of the enhanced image and/or amplify existing noises [2]. In addition, due to the poor and low contrast nature of the acquired image, vagueness and ambiguity are introduced and have led to the increment of uncertainty in the image information. This vagueness in the 
image appears in the form of imprecise boundaries and intensities during image digitization.

Therefore, fuzzy sets theory [6] has been proposed as a problem solving tool between the precision of classical mathematics and the inherent imprecision of the real world. The imprecision possessed by the acquired image can be perceived qualitatively by human reasoning. However, there is no specific quantification to describe the imprecision and thus machine may not understand them. Realizing this limitation to a great extent, fuzzy logic tools empower a machine to mimic human reasoning.

In the image enhancement field, the fuzzy set theory has been widely utilized by other researchers [1,4,7-14]. Pixel property such as gray tone intensity is modeled into a fuzzy set using a membership function. The image is considered as an array of fuzzy singletons having a membership value that denotes the degree of belonging to specific property.

In 2000 H.D. Cheng et.al [7], proposed a novel adaptive direct fuzzy contrast enhancement method, sigmoidal membership function is used to map an image from spatial to fuzzy domain. The resultant image obtained is properly enhanced. When transforming the image from one color space (RGB) to another color space (HSV, HIS, YIQ) hue is unaltered only the intensity and saturation components are changed, as a result gamut problem will occur. So in [8], S.K.Naik tried to keep the transformed values within the range of the RGB space so as to avoid the gamut problem. Although the image is enhanced, the clarity of the enhanced image is not good.

In 2006 Madasu Hanmandlu, and Devendra Jha [4] proposed that Gaussian membership function to fuzzify the image information in spatial domain. They introduce a global contrast intensification operator (GINT), which contains three parameters, viz., intensification parameter, fuzzifier, and the crossover point, for enhancement of color images. They define fuzzy contrast-based quality factor and entropy-based quality factor and the corresponding visual factors for the desired appearance of images. By using the proposed technique, they observed a visible improvement in the image quality for under exposed images, and the entropy of the output image is decreased.

In 2009 Madasu Hanmandlu. et.al [9] presented a new approach for the enhancement of color images using the fuzzy logic technique. To provide an estimate of the underexposed and overexposed regions in the image, an objective measure called exposure has been defined. This measure serves as the dividing line between the underexposed and overexposed regions of the image. For the process of enhancement, The hue, saturation, and intensity (HSV) color space is employed. The hue component is preserved to keep the original color composition intact. For the enhancement of the luminance component of the underexposed image a parametric sigmoid function is used. A power-law operator is used to improve the overexposed region of the image, and the saturation component of HSV is changed through another power-law operator to recover the lost information in the overexposed region.

In 2011 Gang $\mathrm{Li}$ et.al [10] proposed image enhancement operation that used the value of grey entropy in the neighborhood window as parameters to measure the level of current pixel being edge point. This paper described a fuzzy mapping based on translation transformation, which can increase the stability of the algorithm; making use the grey entropy of pixels in neighborhood to judge the level of edge for pixels, the dynamic adaptive selection of central point of neighborhood in fuzzy contrast enhancement was achieved, and can increase the local gray contrast of the image, rich the texture layer of the image ,improve the quality of the image , make it more adaptive for further treatment and analysis.

In 2012 Khairunnisa Hasikin and Nor Ashidi Mat Isa [11] presented a fuzzy grayscale enhancement technique for low contrast image. Most of the developed contrast enhancement techniques improved image quality without considering the non uniform lighting in the image. Here, the fuzzy grayscale image enhancement technique is proposed by maximizing fuzzy measures contained in the image. Then, to enhance the image, membership function is modified by using power-law transformation and saturation operator..

Image enhancement algorithms offer a wide variety of approaches for modifying images to achieve visually acceptable images. The choice of such techniques is a function of the specific task, image content, observer characteristics, and viewing conditions. In this section, there is a survey on various techniques for image enhancement. This paper presents a enhancement technique for low contrast grayscale image. The paper first, transforms the gray scale image from spatial domain to fuzzy domain, then maximizes the fuzzy measures contained in the image by using the membership function that is modified to enhance the image by using power-law transformation. And finally, defuzzification is applied that transforms the gray scale image from modified fuzzy domain back to spatial domain.

The paper is organized as follows: Section III describes the proposed work implementation and algorithm which describes the method that is defined for image enhancement. Section IV Shows experimental results of proposed method and comparison with other image enhancement Methods. Section $\mathrm{V}$ Describes the conclusion and future work of proposed method.

\section{PROPOSED METHOD}

\subsection{Image representation in fuzzy set notation}

An image $\mathrm{X}$ of size $\mathrm{M} * \mathrm{~N}$ having gray levels ranging from Lmin to Lmax can be modeled as an array of fuzzy singletons. Each element in the array is the membership value representing the degree of brightness of the gray level 1 (l= Lmin, Lmin $+1, \ldots$, , Lmax). In the fuzzy set notation, it can be written

$\mu_{i j}=\left(X_{i j}-X_{\min }\right) /\left(X_{\max }-X_{\min }\right)$

Where $\mu_{i, j}$ denotes the degree of brightness possessed by the gray level intensity $x_{i, j}$ of the (i,j)th pixel.

\subsection{Modification of Membership Function}

The goal of the proposed method is to take care of the fuzzy nature of an image and the fuzziness in the definition of the contrast to make the contrast enhancement more adaptive and more effective, and to avoid over-enhancement/underenhancement. So for adaptive fuzzy contrast enhancement fuzzification is applied by Modification of memberships function $\mu_{i, j} \rightarrow \mu_{i, j}$ by using following PAL and KING transformation or the intensification operator (INT), 


$$
\begin{aligned}
& \mu_{i j}^{\prime}=T\left(\mu_{i j}\right) \\
& \mu_{i j}^{\prime}= \begin{cases}2 *\left[\mu_{i j}\right]^{2} & 0 \leq \mu_{i j} \leq \mu_{c} \\
1-2 *\left[1-\mu_{i j}\right]^{2} & \mu_{c}<\mu_{i j} \leq 1\end{cases}
\end{aligned}
$$

It transforms the membership values that are above 0.5 (default value) to much higher values and membership values that lower than 0.5 to much lower values in a nonlinear manner to obtain good enhancement image otherwise show the not enhancement image.

\subsection{Adaptive fuzzy contrast enhancement using defuzzification}

Defuzzification for the generation of new gray levels $X_{i j}^{\prime}$ by the inverse transformation $G^{-1}$.

$$
\begin{aligned}
& X_{i j}^{\prime}=G^{-1}\left(\mu_{i j}^{\prime}\right) \\
& X_{i j}^{\prime}=X_{\min }+\mu_{i j}^{\prime} *\left(X_{\max }-X_{\min }\right)
\end{aligned}
$$

Thus, the final image obtained by the defuzzification process, is the required enhanced image for the input image, $X$ '.

\section{PERFORMANCE ANALYSIS}

The proposed method has been implemented on Intel Core 2 CPU 2GHz using Matlab R2009b. 20 standard images (size: 400x264) obtained from California Institute of Technology database which consist of underexposed and overexposed regions are considered as test images.

The enhanced image is analyzed in terms of its output quality and quantitative analysis such as index of fuzziness (IOF), contrast, peak signal to noise ratio (PSNR) and processing time.

In order to demonstrate the performance of the proposed method, There is a comparison of the experimental results of the proposed approach with other state of the art methods namely fuzzy set theory [1], conventional approach of NINT [6], application of fuzzy IF-THEN rules (fuzzy rule-based) [12], Pal-King method [15] fuzzy quantitative measure [13] and fuzzy local enhancement [14], are widely used in image enhancement.

The enhanced images produced by the proposed methods are presented in Fig. 1 to 4 . For the subjective qualitative analysis of processed image appearance, the test images namely 'Lena, 'plane, 'Baboon' and 'Paper' are shown in these figures. The original images have poor brightness in the underexposed regions and brightness is higher in the overexposed regions.
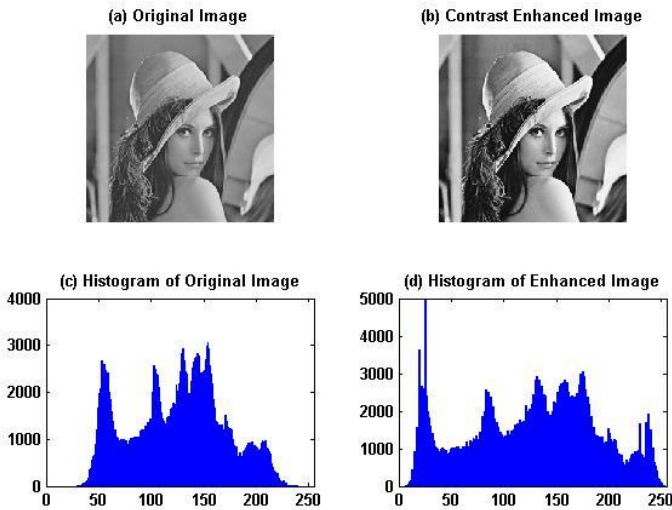

Fig 2: (a) original image (Lena),(b) enhanced image c) Histogram of Original Image (d) histogram of Enhance image
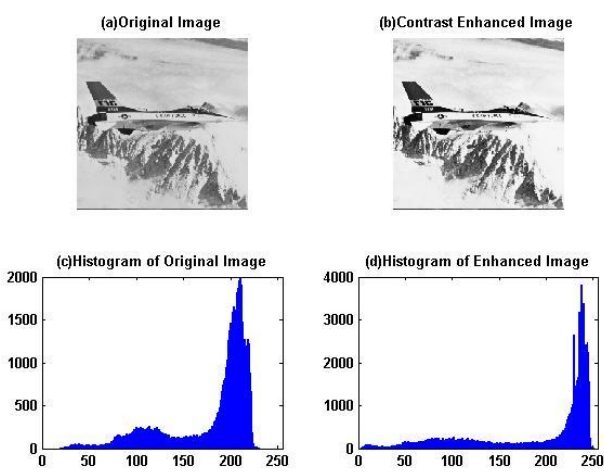

Fig 3: (a) original image (Plane), (b) enhanced image c) Histogram of Original Image (d) histogram of Enhance image
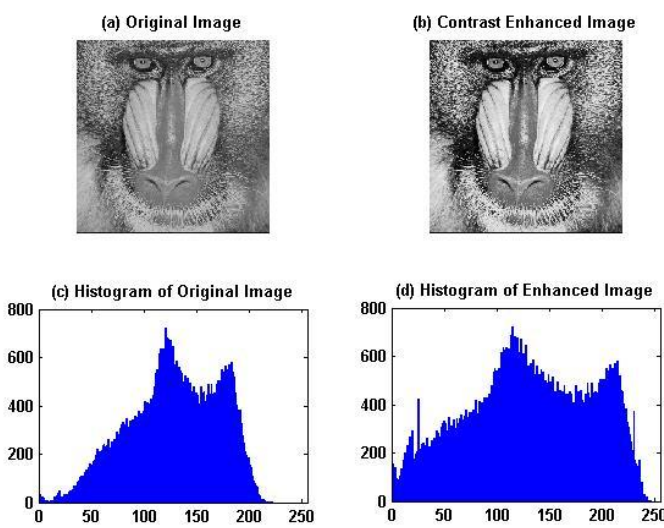

Fig 4: (a) original image (Baboon), (b) enhanced image c) Histogram of Original Image (d) histogram of Enhance image 

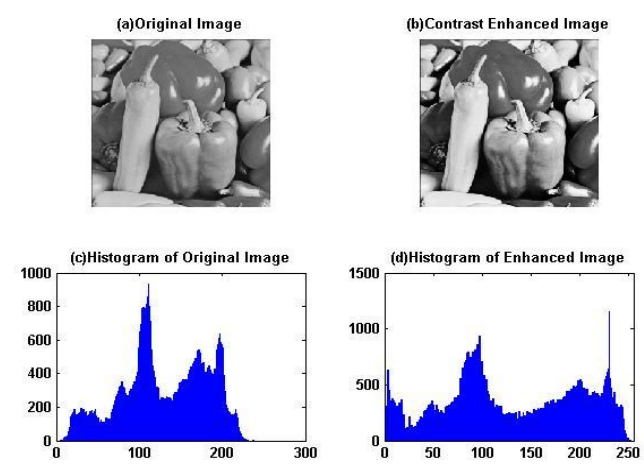

Fig 5: (a) original image (Paper), (b) enhanced image c) Histogram of Original Image (d) histogram of Enhance image

The qualitative analysis presented in the Figures 2 to 5 can be supported by quantitative analysis presented in TABLE I. The average analysis for 20 standard images of proposed method, NINT, fuzzy rule-based, fuzzy quantitative analysis ,Pal King method and fuzzy local enhancement presented in TABLE I are discussed. For each analysis, the best results obtained are made bold.

TABLE I indicates that the proposed method has the best performances in terms of smallest IOF, highest PSNR and obtained good contrast. However, in terms of the average execution time, NINT has the fastest processing time because NINT is less complex and treated the whole image as mixed region without considering overexposed and underexposed regions.

Table 1. Quantitative Enhancement Analyses For 20 Standard Images (Average Values)

\begin{tabular}{|c|c|c|c|c|}
\hline Method\Analysis & $\begin{array}{c}\text { Processing } \\
\text { Time } \\
\text { T(s) }\end{array}$ & IOF & $\begin{array}{c}\text { PSNR } \\
\text { (dB) }\end{array}$ & $\begin{array}{l}\text { Cont } \\
\text { rast }\end{array}$ \\
\hline Proposed Method & 0.051 & 0.451 & 35.21 & $\begin{array}{c}71.71 \\
51\end{array}$ \\
\hline $\begin{array}{c}\text { Fuzzy Set } \\
\text { Enhancement }\end{array}$ & 0.062 & 0.349 & 22.039 & $\begin{array}{c}71.96 \\
9 \\
\end{array}$ \\
\hline NINT & 0.050 & 0.443 & 13.947 & $\begin{array}{c}88.39 \\
1 \\
\end{array}$ \\
\hline Pal- King & 3.251 & 0.572 & 18.92 & 89.1 \\
\hline Fuzzy rule-based & 11.921 & 0.367 & 19.096 & $\begin{array}{c}78.79 \\
3 \\
\end{array}$ \\
\hline $\begin{array}{c}\text { Fuzzy Quantitative } \\
\text { Measure }\end{array}$ & 0.063 & 0.410 & 15.417 & $\begin{array}{c}82.65 \\
4 \\
\end{array}$ \\
\hline $\begin{array}{c}\text { Fuzzy Local } \\
\text { Enhancement }\end{array}$ & 11.163 & 0.584 & 19.063 & $\begin{array}{c}81.92 \\
9\end{array}$ \\
\hline
\end{tabular}

Fig.6 shows the computational time of the proposed method with other enhancement methods . Fig.7 shows comparison graph of the IOF among proposed method and other enhancement methods. Fig. 8 shows comparison graph of the proposed method and other enhancement methods with respect to PSNR Calculation .

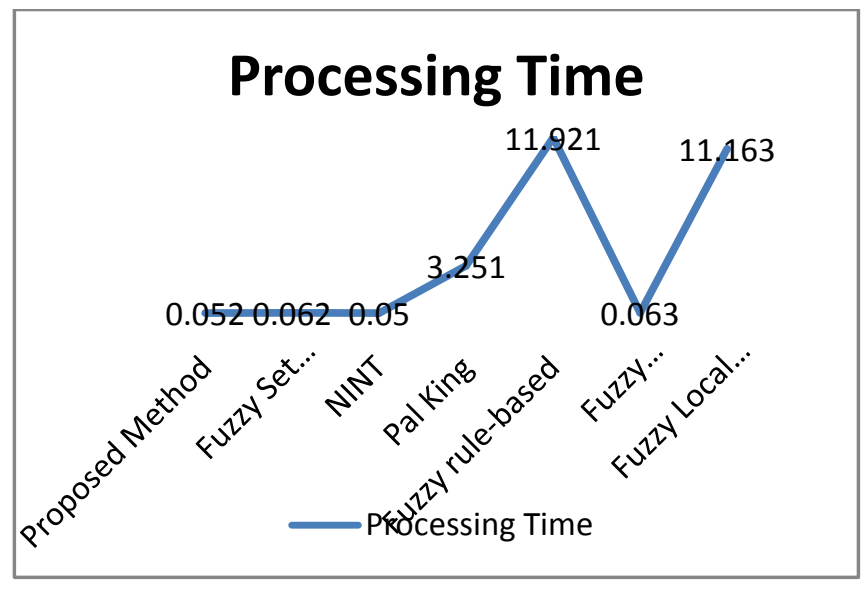

Fig 6: The Execution Time(sec) comparison of the proposed Method with other existing method

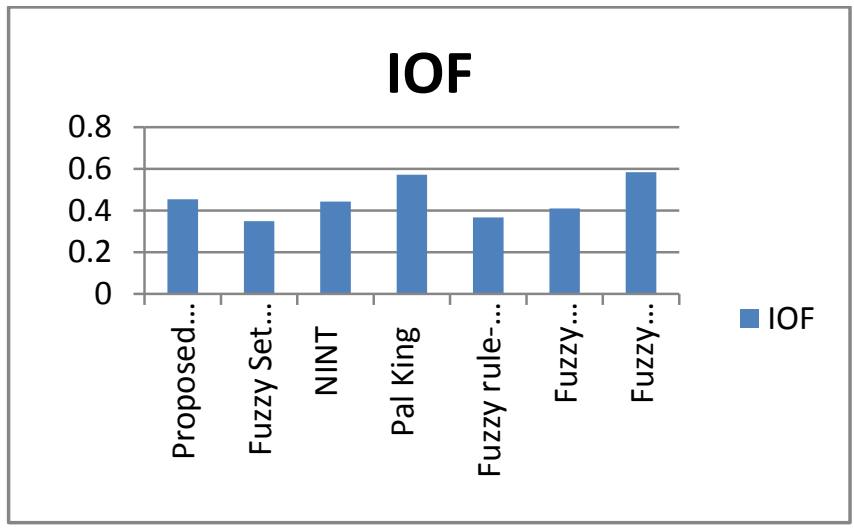

Fig 7:The IOF comparison of the proposed Method with other existing method

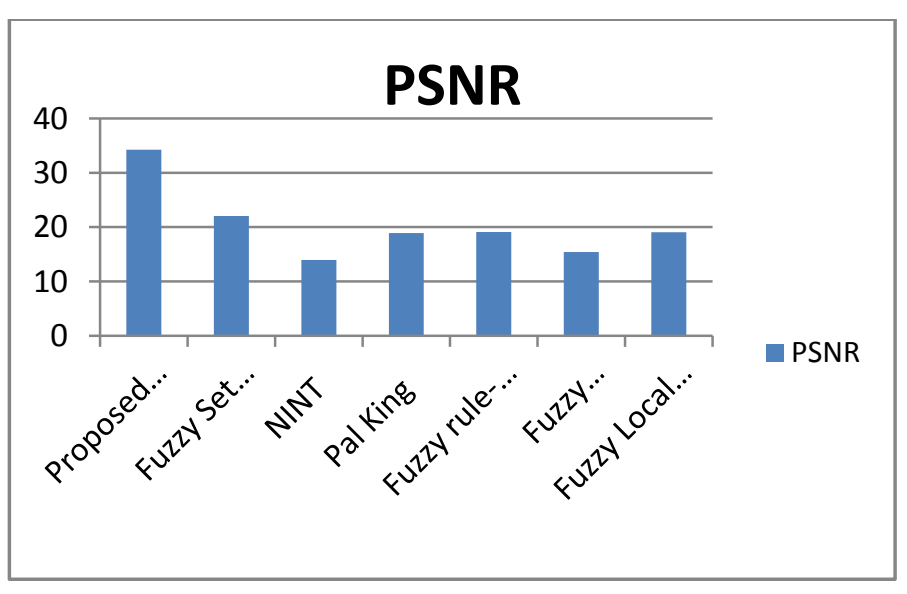

Fig 8:The PSNR comparison of the proposed Method with other existing method

\section{CONCLUSION}

In this paper Pal King Membership function is defined to enhance the image and algorithm is proposed. The proposed algorithm is implemented in MATLAB 7.8. This proposed algorithm is able to overcome the drawbacks of spatial domain methods like thresholding, histogram equalization and frequency domain methods. This algorithm is able to get good contrasted image which increases the brightness of the low contrasted images. This algorithm is tested on different type of images. The experimental result shows that the brightness is increased as compared to previous one. Future work can be 
extended for other images then grayscale images to obtain better result with accuracy.

\section{REFERENCES}

[1] Khairunnisa Hasikin and Nor Ashidi Mat Isa, 2012. "Enhancement of the low contrast image using fuzzy set theory"14th International Conference on Modelling and Simulation, IEEE.

[2] S.S. Bedi1, Rati Khandelwal2," 2013. Various Image Enhancement Techniques- A Critical Review" International Journal of Advanced Research in Computer and Communication Engineering Vol. 2, Issue 3, March 2013

[3] Raman Maini and Himanshu Aggarwal, Mar 2010. "A Comprehensive Review of Image Enhancement Techniques" JOURNAL OF COMPUTING, VOLUME 2, ISSUE 3, ISSN 2151-9617.

[4] M. Hanmandlu and D. Jha, 2006. "An Optimal Fuzzy System for Color Image Enhancement," Image Processing, IEEE Transactions on, vol. 15, pp. 29562966

[5] Manpreet Kaur, Jasdeep Kaur, Jappreet Kaur, 2011. ” Survey of Contrast Enhancement Techniques based on Histogram Equalization" (IJACSA) International Journal of Advanced Computer Science and Applications, Vol. 2, No. 7.

[6] L. A. Zadeh, 1973. "Outline of a New Approach to the Analysis of Complex Systems and Decision Processes," Systems, Man and Cybernetics, IEEE Transactions on, vol. SMC-3, pp. 28-44.

[7] H.D. Cheng, Huijuan Xu, 2000 "A novel fuzzy logic approach to contrast enhancement", Pattern Recognition , $33,2000,809-819$.
[8] S.K. Naik, C.A. Murthy, 2003 "Hue-preserving color image enhancement without gamut problem", IEEE Trans. Image Process.,12, 2003, 1591-1598.

[9] Madasu Hanmandlu, Senior Member, IEEE, Om Prakash Verma, Nukala Krishna Kumar, and Muralidhar Kulkarni, Aug 2009 "A Novel Optimal Fuzzy System for Color Image Enhancement Using Bacterial Foraging”, IEEE transactions on instrumentation and measurement, vol. 58 , no. 8 .

[10] Gang Li, Yala Tong,Xinping Xiao, 2011 “Adaptive Fuzzy Enhancement Algorithm of Surface Image based on Local Discrimination via Grey Entropy", Elsevier Procedia Engineering 15 (2011) 1590 - 1594.

[11] Khairunnisa Hasikin and Nor Ashidi Mat Isa, 2012 "Enhancement of the low contrast image using fuzzy set theory"14th International Conference on Modelling and Simulation, IEEE.

[12] X.-Y. Wang, T. Wang, and J. Bu, 2011 "Color image segmentation using pixel wise support vector machine classification," Pattern Recognition, vol. 44, pp. 777-787.

[13] D.-1. Peng and A.-k. Xue, 2005 "Degraded image enhancement with applications in robot vision," in Systems, Man and Cybernetics, IEEE International Conference on, 2005, pp. 1837-1842 Vol. 2.

[14] E. E. Kerre and M. Nachtegael,2000 Fuzzy techniques in image processing: Physica-Verlag.

[15] Pal S K, King R A. 1981 "Image enhancement using smoothing with fuzzy sets". IEEE Trans. Systems, Man \& Cybernetics, 11(7): 494-501. 